Pacific Journal of Mathematic 


\title{
ON THE UNIFORM CONVERGENCE OF A CERTAIN EIGENFUNCTION SERIES
}

\author{
L. I. Mishoe and G. C. Ford
}

1. Introduction. In the attempt to solve certain problems in mathematical physics, such as diffraction of an arbitrary pulse by a wedge as considered by Irvin Kay [1], one encounters a hyperbolic differential equation of the type

$$
u_{x x}-q(x) u=u_{x t}-p(x) u_{t}
$$

where $u(x, t)$ must satisfy the boundary conditions $u(1, t)=u(0, t)=0$ and $u(x, 0)=F(x)$. In attempting to solve equation (a) by separation of variables, one is led to the consideration of expanding an arbitrary function $F(x)$ in terms of the eigenfunctions $u_{n}(x)$ of the equation

$$
u^{\prime \prime}+q(x) u+\lambda\left(p(x) u-u^{\prime}\right)=0
$$

satisfying the boundary conditions $u(0)=u(1)=0$.

In the previous paper [2] by B. Friedman and L. I. Mishoe, it was proved that a function $F(x)$ of bounded variation for $0 \leqq x \leqq 1$ could be expanded in terms of the eigenfunctions $u_{n}(x)$ of the system $u^{\prime \prime}+q u+$ $\lambda\left(p u-u^{\prime}\right)=0, u(0)=u(1)=0$, provided $F\left(0^{+}\right)+F\left(1^{-}\right) \exp \left(-\int_{0}^{1} p d t\right)=0$. However, the question of uniform convergence of the series $\sum_{-\infty}^{\infty} a_{n} u_{n}(x)$ to $F(x)$ was not considered. In this paper we establish sufficient conditions for the series $\sum_{-\infty}^{\infty} a_{n} u_{n}(x)$ to converge uniformly to $F(x)$ for $0<x<1$.

The following theorem has already been proved [2]:

THEOREM 1. Let $F(x)$ be a function of bounded variation for $0 \leqq x \leqq 1$. Let $u_{n}(x)$ be the eigenfunctions of the system

$$
(A+\lambda B) u=0 ; \quad u(0)=u(1)=0,
$$

where $A$ is the operator $d^{2} / d x^{2}+q(x)$, and where $B$ is the operator $-d / d x$ $+p(x)$.

Let $q(x)$ be continuous and $p(x)$ have a continuous second derivative. Furthermore, let $v_{n}(x)$ be the eigenfunctions of the system adjoint to (1). If

Received February 2, 1955. The research in this document has been made possible through a grant to Morgan State College by the National Science Foundation, Washington, D. C. Research Grant NSF-G 1224, November 1954. 


$$
F\left(0^{+}\right)+F\left(1^{-}\right) \exp \left(-\int_{0}^{1} p(t) d t\right)=0,
$$

then the series

$$
\sum_{-\infty}^{\infty} a_{n} u_{n}(x)
$$

where

$$
a_{n}=\int_{0}^{1} F(\xi)\left[\frac{p(\xi) v_{n}(\xi)+v_{n}^{\prime}(\xi)}{C^{\prime}\left(\lambda_{n}\right)}\right] d \xi
$$

and where the Wronskian $\omega(x)$ of the two independent solutions $u_{1}(x)$ and $u_{2}(x)$ has the form

$$
\omega(x)=u_{1} u_{2}^{\prime}-u_{2} u_{1}^{\prime}=C(\lambda) e^{\lambda x}
$$

with

$$
C(\lambda)=\lambda^{-1} \exp \left(-\int_{0}^{1} p(t) d t\right)-\exp \left(-\lambda+\int_{0}^{1} p(t) d t\right)+O\left(\lambda^{-2}\right),
$$

converges to $F(x)$ at every point where $F(x)$ is continuous in $0<x<1$. At all other points, the series converges to $\frac{1}{2}(F(x+0)+F(x-0))$. If $F(x)$ does not satisfy the boundary conditions (2), then the series (3) converges to

$$
\frac{1}{2}\left[F(x+0)+F(x-0)-\left\{F\left(0^{+}\right)+F\left(1^{-}\right) \exp \left(-\int_{0}^{1} p(t) d t\right)\right\} \exp \left(\int_{0}^{x} p(t) d t\right)\right] \text {. }
$$

In this paper, we prove:

THEOREM 2. If $F^{\prime \prime}(x)$ exists and is of bounded variation for $0 \leqq x \leqq 1$, then a sufficient condition for the series $\sum_{-\infty}^{\infty} a_{n} u_{n}(x)$ to converge uniformly to $F(x)$ for $0<x<1$ is that $F(0)=F(1)=0$.

2. An asymptotic form for $C^{\prime}\left(\lambda_{n}\right)$. Using (5) and the boundary conditions $u(0)=u(1)=0$ and $u^{\prime}(0)=u^{\prime}(1)=1$, we have

$$
C(\lambda)=e^{-\lambda} u_{1}(1, \lambda) \text {. }
$$

Then it follows that

$$
C^{\prime}(\lambda)=\frac{d}{d \lambda} C(\lambda)=-C(\lambda)+\frac{1}{2} e^{-\lambda} u_{1}(1, \lambda)+e^{-\lambda / 2} \frac{d}{d \lambda} w_{1}(x, \lambda) \quad \text { at } x=1
$$

where

$$
u_{1}=e^{\lambda x / 2} w_{1}
$$


Now (10) transforms the equation $(A+\lambda B) u=0$ into $w_{1}^{\prime \prime}+\left(q+\lambda p-\frac{\lambda^{2}}{4}\right) w_{1}=0$.

It can be verified [2] that $w_{1}$ satisfies the equation

$$
\left.w_{1}=\frac{\sinh R(0, x)}{[r(x) r(0)]^{1 / 2}}-\int_{0}^{x} \sinh R(\xi, t) g(\xi) r(\xi)\right]^{1 / 2} g(\xi) w_{1}(\xi) d \xi
$$

where

$$
g(x)=p^{2}+\frac{p^{\prime \prime}}{\lambda-2 p}+\frac{3 p^{\prime 2}}{(\lambda-2 p)^{2}}+q
$$

and

$$
r(x)=\frac{\lambda}{2}-p(x), \quad R(\xi, x)=\int_{\xi}^{x} r(t) d t
$$

We note that $g(x)$ and $g^{\prime}(x)=\frac{d}{d \lambda} g(x)$ are bounded for $|\lambda|$ sufficiently large. Also, if in (11) we make the substitution

$$
w_{1}=\lambda^{-1} \exp \left(\frac{1}{2}|\sigma| x\right) Z_{1}(x)
$$

where $\sigma=\mathscr{R} \lambda$, we note that $Z_{1}(x)$ is bounded [2] for $|\lambda|$ sufficiently large. Differentiating (11) with respect to $\lambda$, we obtain

$$
\begin{aligned}
w_{1}^{\prime} & =\frac{x \cosh R(0, x)}{2[r(x) r(0)]^{1 / 2}}-\frac{[r(x)+r(0)] \sinh R(0, x)}{4[r(x) r(0)]^{3 / 2}} \\
& -\int_{0}^{x} \frac{\sinh R(\xi, x)}{[r(x) r(\xi)]^{1 / 2}} g(\xi) w_{1}^{\prime}(\xi) d \xi-\int_{0}^{x} \frac{\sinh R(\xi, x)}{[r(x) r(\xi)]^{1 / 2}} g^{\prime}(\xi) w_{1}(\xi) d \xi \\
& -\int_{0}^{x}(x-\xi) \frac{\cosh R(\xi, x)}{[r(x) r(\xi)]^{1 / 2}} g(\xi) w_{1}(\xi) d \xi \\
& +\frac{1}{4} \int_{0}^{x} \frac{[r(x)+r(\xi)] \sinh R(\xi, x)}{[r(x) r(\xi)]^{3 / 2}} g(\xi) w_{1}(\xi) d \xi
\end{aligned}
$$

If we substitute

$$
w_{1}=\lambda^{-1} \rho(x) \exp \left(\left.\frac{1}{2}\right|_{\sigma} \mid x\right)
$$

we obtain that

$$
\begin{aligned}
\rho(x) & =\frac{\lambda x \exp \left(-\frac{1}{2}|\sigma| x\right) \cosh R(0, x)}{[r(x) r(0)]^{1 / 2}} \\
& -\frac{\lambda[r(x)+r(0)] \exp \left(-\frac{1}{2}|\sigma| x\right) \sinh R(0, x)}{4[r(x) r(0)]^{3 / 2}}
\end{aligned}
$$




$$
\begin{aligned}
& -\int_{0}^{x} \frac{\exp \left[-\frac{1}{2}|\sigma|(x-\xi)\right] \sinh R(\xi, x)}{[r(x) r(\xi)]^{1 / 2}} g(\xi) \rho(\xi) d \xi \\
& -\int_{0}^{x} \frac{\exp \left[-\frac{1}{2}|\sigma|(x-\xi)\right] \sinh R(\xi, x)}{[r(x) r(\xi)]^{1 / 2}} g^{\prime}(\xi) Z_{1}(\xi) d \xi \\
& -\int_{0}^{x} \frac{1}{2}(x-\xi) \frac{\exp \left[-\frac{1}{2}|\sigma|(x-\xi)\right] \cosh R(\xi, x)}{[r(x) r(\xi)]^{1 / 2}} g(\xi) Z_{1}(\xi) d \xi \\
& +\int_{0}^{x} \frac{[r(x)+r(\xi)] \exp \left[-\frac{1}{2}|\sigma|(x-\xi)\right] \sinh R(\xi, x)}{4[r(x) r(\xi)]^{3 / 2}} g(\xi) Z_{1}(\xi) d \xi
\end{aligned}
$$

where $g^{\prime}(\xi)=\frac{d}{d \lambda} g(\xi)$. Now $\lambda[r(x) r(\xi)]^{-1 / 2}$ and hence $\lambda[r(x)+r(\xi)][r(x) r(\xi)]^{-3 / 2}$ are both bounded by some constant $C$ as $|\lambda| \rightarrow \infty$. Also, exp $\left[-\frac{1}{2}|\sigma|(x-\xi)\right]$ $\times \cosh R(\xi, x)$, and $\exp \left[-\frac{1}{2}|\sigma|(x-\xi)\right] \sinh R(\xi, x)$ are both bounded by some constant $C$ as $|\lambda| \rightarrow \infty$ and $0 \leqq \xi \leqq x$. Using these results we obtain from equation (17) that

$$
\begin{aligned}
|\rho(x)| & \leqq 2 C^{2}+\frac{C^{2}}{|\lambda|} \int_{0}^{x}|g(\xi) \rho(\xi)| d \xi+\frac{C^{2}}{|\lambda|} \int_{0}^{x}\left|g^{\prime}(\xi) Z_{1}(\xi)\right| d \xi \\
& +\frac{C^{2}}{|\lambda|} \int_{0}^{x} \frac{1}{2}|x-\xi|\left|g(\xi) Z_{1}(\xi)\right| d \xi+\frac{C^{2}}{|\lambda|} \int_{0}^{x}\left|g(\xi) Z_{1}(\xi)\right| d \xi .
\end{aligned}
$$

If we set $\mu(\lambda)$ equal to the maximum of $|\rho(x)|$ in $0 \leqq x \leqq 1$, then we certainly have that

$$
\mu \leqq \frac{2 C^{2}}{1-\frac{C^{2}}{|\lambda|} \int_{0}^{x}|g(\xi)| d \xi}+\frac{C^{2} \int_{0}^{x}\left(\left|g^{\prime}(\xi) Z_{1}(\xi)\right|+\frac{1}{2}|x-\xi|\left|g(\xi) Z_{1}(\xi)\right|+\left|g(\xi) Z_{1}(\xi)\right|\right) d \xi}{1-\frac{C^{2}}{|\lambda|} \int_{0}^{x}|g(\xi)| d \xi} .
$$

Therefore, $\mu$, and consequently $\rho(x)$ are bounded as $|\lambda| \rightarrow \infty$. Rewrite equation (15) as follows:

$$
\begin{aligned}
& w_{1}^{\prime}(x, \lambda)=\frac{x \cosh R(0, x)}{2[r(x) r(0)]^{1 / 2}}-\frac{[r(x)+r(0)] \sinh R(0, x)}{4[r(x) r(0)]^{3 / 2}} \\
& -\lambda^{-1} \exp \left(\frac{1}{2}|\sigma| x\right) \int_{0}^{x} \frac{\exp \left[-\frac{1}{2}|\sigma|(x-\xi)\right] \sinh R(\xi, x)}{[r(x) r(\xi)]^{1 / 2}} g(\xi) \rho(\xi) d \xi \\
& -\lambda^{-1} \exp \left(\frac{1}{2}|\sigma| x\right) \int_{0}^{x} \frac{\exp \left[-\frac{1}{2}|\sigma|(x-\xi)\right] \sinh R(\xi, x)}{[r(x) r(\xi)]^{1 / 2}} g(\xi) Z_{1}(\xi) d \xi \\
& -\lambda^{-1} \exp \left(\frac{1}{2}|\sigma| x\right) \int_{0}^{x}(x-\xi) \frac{\exp \left[-\frac{1}{2}|\sigma|(x-\xi)\right] \sinh R(\xi, x)}{2[r(x) r(\xi)]^{1 / 2}} g(\xi) Z_{1}(\xi) d \xi \\
& +\lambda^{-1} \exp \left(\frac{1}{2}|\sigma| x\right) \int_{0}^{x} \frac{\exp \left[-\frac{1}{2}|\sigma|(x-\xi)\right][r(x)+r(\xi)] \sinh R(\xi, x)}{4[r(x) r(\xi)]^{3 / 2}} g(\xi) Z_{1}(\xi) d \xi \text {. }
\end{aligned}
$$


The above four integrals are all at least $O\left(\lambda^{-2} \exp \left[\frac{1}{2}|\sigma| x\right]\right)$. Also,

$$
[r(x) r(0)]^{-1 / 2} \doteq \lambda^{-1}+O\left(\lambda^{-2}\right),
$$

and

$$
[r(x)]^{-1 / 2}[r(0)]^{-3 / 2}=\lambda^{-2}+O\left(\lambda^{-3}\right)
$$

So it follows that

(20) $w_{1}^{\prime}(1, \lambda)=\lambda^{-1} \cosh \left(\int_{0}^{1} r(t) d t\right)+O\left(\lambda^{-2} \exp \left[\frac{1}{2}|\sigma|\right]\right)-\frac{\lambda^{-2}}{2} \sinh \left(\int_{0}^{1} r(t) d t\right)$

$$
+O\left(\lambda^{-3} \exp \left[\frac{1}{2}|\sigma|\right]\right) \text {. }
$$

Using this result for $w_{1}^{\prime}(1, \lambda)$ in equation (9), we have, for $\mathscr{R} \lambda>0$,

$$
\begin{aligned}
C^{\prime}(\lambda) & =\lambda^{-1}\left[\exp \left(-\lambda+\int_{0}^{1} p(t) d t\right)\right] \\
& -\frac{1}{2} \lambda^{-2}\left[\exp \left(-\int_{0}^{1} p(t) d t\right)-\exp \left(-\lambda+\int_{0}^{1} p(t) d t\right)\right]+O\left(\lambda^{-3}\right)+O\left(\lambda^{-2}\right),
\end{aligned}
$$

and for $\mathscr{R} \lambda<0$,

$$
\begin{aligned}
C^{\prime}(\lambda) & =\lambda^{-1}\left[\exp \left(-\lambda+\int_{0}^{1} p(t) d t\right)\right] \\
& -\frac{1}{2} \lambda^{-2}\left[\exp \left(-\int_{0}^{1} p(t) d t\right)-\exp \left(-\lambda+\int_{0}^{1} p(t) d t\right)\right]+O\left(\lambda^{-3} e^{\lambda}\right) .
\end{aligned}
$$

3. Distribution of the eigenvalues. Since by [2]

$$
\begin{array}{rlr}
C(\lambda) & =\lambda^{-1} \exp [-\lambda a]\left(\exp \left[-\int_{a}^{b} p(t) d t\right]-\exp \left[-\lambda(b-a)+\int_{a}^{b} p(t) d t\right]+O\left(\lambda^{-1}\right)\right) \\
& =\lambda^{-1} \exp [-\lambda a] C_{1}(\lambda) \\
C(\lambda) & =\lambda^{-1} \exp [-\lambda b]\left(\exp \left[-\lambda(a-b)-\int_{a}^{b} p(t) d t\right]-\exp \left[\int_{a}^{b} p(t) d t\right]+O\left(\lambda^{-1}\right)\right) \\
& =\lambda^{-1} \exp [-\lambda b] C_{2}(\lambda) & \text { for } \mathscr{R} \lambda \geqq 0,
\end{array}
$$

and where $a$ and $b$ equal 0 and 1 respectively.

The condition that $\lambda$ be an eigenvalue is that $C(\lambda)$ and hence either $C_{1}(\lambda)$ or $C_{2}(\lambda)$ be zero. Equating $C_{1}(\lambda)$ to zero we obtain

$$
\begin{aligned}
\exp \left[-\lambda(b-a)+\int_{a}^{b} p(t) d t\right] & =\exp \left[-\int_{a}^{b} p(t) d t\right]+O\left(\lambda^{-1}\right) \\
& =\exp \left[-\int_{a}^{b} p(t) d t\right]\left(1+O\left(\lambda^{-1}\right)\right) .
\end{aligned}
$$


By taking the logarithm of both sides of the above equation (23) and expanding the term $\log \left(1+O\left(\lambda^{-1}\right)\right)$ we obtain that the large eigenvalues satisfy the equation

$$
-\lambda_{n}=-2 \int_{0}^{1} p(t) d t+2 n \pi i+O\left(\lambda_{n}^{-1}\right), \quad n= \pm N, \pm N+1, \cdots .
$$

Hence the eigenvalues with positive real parts, if they exist, are given by

$$
\lambda_{n}=2 n \pi i+2 \int_{0}^{1} p(t) d t+O\left(\frac{1}{n}\right)
$$

The equation $C_{2}(\lambda)=0$ leads to the same result for those eigenvalues with negative real parts. Consequently, all the eigenvalues are represented by equation (24).

4. On the uniform convergence of series (3). Consider equation (4). In [2] it was shown that

$$
B^{*} V_{1}(x)=\exp \left[-\lambda x+\int_{0}^{x} p(t) d t\right]+\Omega_{1},
$$

where

$$
\Omega_{1}= \begin{cases}O\left(\lambda^{-2}\right)+O\left(\lambda^{-1} \exp [-\lambda x]\right) & \text { for } \mathscr{R} \lambda \geqq 0 \\ O\left(\lambda^{-1} \exp [-\lambda x]\right) & \text { for } \mathscr{R} \lambda \leqq 0 .\end{cases}
$$

Similarly,

$$
B^{*} V_{2}(x)=\exp \left[-\lambda x+\int_{1}^{x} p(t) d t\right]+\Omega_{2}
$$

where

$$
\Omega_{2}= \begin{cases}O\left(\lambda^{-1} \exp [-\lambda x]\right) & \text { for } \mathscr{R} \lambda \geqq 0 \\ O\left(\lambda^{-1} \exp [-\lambda x]\right)+O\left(\lambda^{-1} \exp [-\lambda b]\right) & \text { for } \mathscr{R} \lambda \leqq 0 .\end{cases}
$$

Also from [2], we have that

$$
\left.u_{1}(x)=\lambda^{-1}\left\{\exp \left[\lambda x-\int_{0}^{x} p(t) d t\right]-\exp \int_{0}^{x} p(t) d t\right]\right\}+O\left(\lambda^{-2} e^{\lambda x}\right)
$$

and

$$
\left.u_{2}(x)=\lambda^{-1}\left\{\exp \left[\lambda(x-1)-\int_{1}^{x} p(t) d t\right]-\exp \int_{1}^{x} p(t) d t\right]\right\}+O\left(\lambda^{-2}\right)
$$

Using equations (26) and (27), for $\mathscr{R} \lambda>0$, we have 


$$
\begin{aligned}
a_{n} u_{1}= & u_{1} \int_{0}^{1} F(\xi) \frac{B^{*} V_{2}(\xi)}{C^{\prime}\left(\lambda_{n}\right)} d \xi \\
= & O\left(\frac{e^{\lambda_{n}} x}{\lambda_{n}}\right) \int_{0}^{1} \frac{F(\xi)\left[\exp \left(-\lambda_{n} \xi+\int_{1}^{\xi} p(t) d t\right)\right] d \xi}{C^{\prime}\left(\lambda_{n}\right)} \\
& +O\left(\lambda_{n}^{-1} e^{\lambda_{n}} x\right) \int_{0}^{1} \frac{F(\xi) O\left(\lambda_{n}^{-1} e^{-\lambda_{n} \xi}\right)}{C^{\prime}\left(\lambda_{n}\right)} d \xi \\
= & \lambda_{n} \int_{0}^{1} \frac{F(\xi) \exp \left(-\lambda_{n} \xi+\int_{1}^{\xi} p(t) d t\right)}{C^{\prime}\left(\lambda_{n}\right)} d \xi+\frac{A}{\lambda_{n} \int_{0}^{1} F(\xi) O\left(\lambda_{n}^{-1} e^{-\lambda_{n} \xi}\right) d \xi} C^{\prime}\left(\lambda_{n}\right)
\end{aligned}
$$

where $A$ is bounded.

By equation (22), $C^{\prime}\left(\lambda_{n}\right)=O\left(\lambda_{n}^{-1}\right)$, therefore $\frac{1}{C^{\prime}\left(\lambda_{n}\right)}=O\left(\lambda_{n}\right)$.

Hence

$$
a_{n} u_{1}=B_{n} \int_{0}^{1} F(\xi) \exp \left(-\lambda_{n} \xi+\int_{0}^{\xi} x(t) d t\right) d \xi+B_{n} \int_{0}^{1} F(\xi) O\left(\lambda_{n}^{-1} e^{-\lambda_{n} \xi}\right) d \xi
$$

where $B_{n}=\lambda^{-1} O\left(\lambda_{n}\right) A$ is also bounded. Using equation (26) for $\mathscr{R} i>0$, and observing that $O\left(\lambda^{-1} \exp \left(-\lambda_{n} \xi\right)\right)$ is the indefinite integral of a bounded function, it can be easily shown that

$$
\int_{0}^{1} F(\xi) O\left(\lambda_{n}^{-1} e^{-\lambda_{n} \xi}\right) d \xi=O\left(\lambda_{n}^{-2}\right) .
$$

Consider now the first integral in equation (29). Setting $H(\xi)=$ $F(\xi) \exp \left(\int_{1}^{\xi} p(t) d t\right)$ and integrating by parts, we obtain

$$
\begin{aligned}
\int_{0}^{1} H(\xi) \exp \left(-\lambda_{n} \xi\right) d \xi= & \left.-\lambda_{n}^{-1} H(\xi) \exp \left(-\lambda_{n} \xi\right)\right]_{0}^{1} \\
& +\int_{0}^{1} \lambda_{n}^{-1} H^{\prime}(\xi) \exp \left(-\lambda_{n} \xi\right) d \xi .
\end{aligned}
$$

Since $F(1)=F(0)=0$, then $H(1)=H(0)=0$, and the first term on the right hand side of equation (31) vanishes.

Now

$$
H^{\prime}(\xi)=p(\xi) F(\xi) \exp \left(\int_{1}^{\xi} p(t) d t\right)+F^{\prime}(\xi) \exp \left(\int_{1}^{\xi} p(t) d t\right) .
$$

$F^{\prime}(\xi)$ is of bounded variation on $(0,1)$ and $p^{\prime}(\xi)$ is continuous on $(0,1)$. Therefore, $H^{\prime}(\xi)$ is of bounded variation on $(0,1)$. Hence,

$$
H^{\prime}(\xi)=\varphi_{1}(\xi)-\varphi_{2}(\xi)
$$


where $\varphi_{1}(\xi)$ and $\varphi_{2}(\xi)$ are two bounded, positive, monotone functions, either both nonincreasing or both nondecreasing. Now $\lambda_{n}^{-1} \exp \left(-\lambda_{n} \xi\right)$ is bounded and integrable for $0 \leqq \xi \leqq 1$. Assume $\varphi_{1}(\xi)$ to be a monotone decreasing function, then

$$
\begin{aligned}
& \int_{0}^{1} \lambda_{n}^{-1} H^{\prime}(\xi) \exp \left(-\lambda_{n} \xi\right) d \xi \\
& \quad=\varphi_{1}(0) \int_{0}^{\xi_{0}} \lambda_{n}^{-1} \exp \left(-\lambda_{n}\right) d \xi-\varphi_{2}(0) \int_{0}^{\xi_{1}} \lambda_{n}^{-1} \exp \left(-\lambda_{n} \xi\right) d \xi=O\left(\lambda_{n}^{-2}\right)
\end{aligned}
$$

where $\xi_{0}$ and $\xi_{1}$ are on the interval $(0,1)$.

Combining the results of (30) and (33) we have

$$
\sum_{-\infty}^{\infty} a_{n} u_{n}(x)=\sum_{-\infty}^{-(N+1)} O\left(\lambda_{n}^{-2}\right)+\sum_{-N}^{N-1} a_{n} u_{n}(x)+\sum_{N}^{\infty} O\left(\lambda_{n}^{-2}\right)
$$

where $\sum_{-N}^{N-1} a_{n} u_{n}(x)$ is finite for $0<x<1$. From (24) it is clear that $\lambda_{n}=O(n)$ for $n= \pm N, \pm N+1, \cdots$ Therefore

$$
\sum_{-\infty}^{\infty} a_{n} u_{n}(x)=\sum_{-\infty}^{-(N+1)} \frac{O(1)}{n^{2}}+\sum_{-N}^{N-1} a_{n} u_{n}(x)+\sum_{N}^{\infty} \frac{O(1)}{n^{2}}
$$

where $O(1)$ is a bounded function.

Since $\left|\frac{O(1)}{n^{2}}\right| \leqq \frac{M}{n^{2}}, M>0$ and the series $M \sum_{N}^{\infty} \frac{1}{n^{2}}$ converges, it is clear that $\sum_{-\infty}^{\infty} a_{n} u_{n}(x)$ converges uniformly to $F(x)$ for $0<x<1$. And our theorem is proved.

We note, however, that while Theorem 2 is sufficient, it is not a necessary condition for uniform convergence. For suppose $F(0)$ and $F(1)$ differ from zero, then by equations (31) and (33) we have $\sum_{-\infty}^{\infty} a_{n} u_{n}=\sum_{-\infty}^{\infty} O\left(\begin{array}{l}1 \\ n\end{array}\right)$ which may or may not converge uniformly.

In fact, a necessary and sufficient condition for the uniform convergence of this series does not seem to be known.

The authors wish to thank Professor Bernard Friedman and Mr. Bertram Levy of New York University, and Miss Noel Cousins of Morgan State College for their assistance in preparing this paper for publication.

\section{REFERENCES}

1. Irvin Kay, Diffraction of an arbitrary pulse by a wedge, Mathematics Research Group, New York University, Report No. EM-43.

2. B. Friedman and L. I. Mishoe, Eigenfunction expansions associated with a non-selfadjoint differential equation, Pacific J. Math., 6 (1956), 249-270. 


\section{PACIFIC JOURNAL OF MATHEMATICS}

EDITORS

\author{
H. L. Royden \\ Stanford University \\ Stanford, California \\ E. Hewite \\ University of Washington \\ Seattle 5 , Washington
}

\author{
R. P. Dilworth \\ California Institute of Technology \\ Pasadena 4, California
}

\author{
A. Horn* \\ University of California \\ Los Angeles 24, California
}

\section{ASSOCIATE EDITORS}
E. F. BECKENBACH
M. HALL
M. S. KNEBELMAN
J. J. STOKER
C. E. BURGESS
P. R. HALMOS
I. NIVEN
V. GANAPATHY IYER
T. G. OSTROM
G. SZEKERES
H. BUSEMANN
R. D. JAMES
M. M. SCHIFFER
F. WOLF
H. FEDERER

\section{SPONSORS}

\author{
UNIVERSITY OF BRITISH COLUMBIA \\ CALIFORNIA INSTITUTE OF TECHNOLOGY \\ UNIVERSITY OF CALIFORNIA \\ CALIFORNIA RESEARCH CORPORATION \\ MONTANA STATE UNIVERSITY \\ UNIVERSITY OF NEVADA \\ OREGON STATE COLLEGE \\ UNIVERSITY OF OREGON \\ UNIVERSITY OF SOUTHERN CALIFORNIA
}

\author{
STANFORD UNIVERSITY \\ UNIVERSITY OF UTAH \\ WASHINGTON STATE COLLEGE \\ UNIVERSITY OF WASHINGTON \\ AMERICAN MATHEMATICAL SOCIETY \\ HUGHES AIRCRAFT COMPANY
}

Mathematical papers intended for publication in the Pacific Journal of Mathematics should be typewritten (double spaced), and the author should keep a complete copy. Manuscripts may be sent to any of the editors. Manuscripts intended for the outgoing editors should be sent to their successors. All other communications to the editors should be addressed to the managing editor, Alfred Horn at the University of California, Los Angeles 24, California.

50 reprints of each article are furnished free of charge; additional copies may be obtained at cost in multiples of 50 .

The Pacific Journal of Mathematics is published quarterly, in March, June, September, and December. The price per volume (4 numbers) is $\$ 12.00$; single issues, $\$ 3.50$. Back numbers are available. Special price to individual faculty members of supporting institutions and to individual members of the American Mathematical Society: $\$ 4.00$ per volume; single issues, $\$ 1.25$.

Subscriptions, orders for back numbers, and changes of address should be sent to Pacific Journal of Mathematics, c/o University of California Press, Berkeley 4, California.

Printed at Kokusai Bunken Insatsusha (International Academic Printing Co., Ltd.), No. 10, 1-chome, Fujimi-cho, Chiyoda-ku, Tokyo, Japan.

* During the absence of E. G. Straus.

PUBLISHED BY PACIFIC JOURNAL OF MATHEMATICS, A NON-PROFIT CORPORATION COPYRIGHT 1956 BY PACIFIC JOURNAL OF MATHEMATICS 


\section{Pacific Journal of Mathematics}

\section{Vol. 6, No. $2 \quad$ December, 1956}

Louis Auslander, Remark on the use of forms in variational calculations .......................................... 209

Hubert Spence Butts, Jr. and Henry B. Mann, Corresponding residue systems in algebraic number fields ........................ 211

L. Carlitz and John Herbert Hodges, Distribution of matrices in a finite field............................................

Paul Civin and Bertram Yood, Invariant functionals ............... 231

David James Dickinson, Henry Pollak and G. H. Wannier, On a class of polynomials orthogonal over a denumerable set .................

Bernard Friedman and Luna Mishoe, Eigenfunction expansions associated with a non-self-adjoint differential equation ....................

Luna Mishoe and G. C. Ford, On the uniform convergence of a certain eigenfunction series .............................. 271

John W. Green, Mean values of harmonic functions on homothetic curves...........................................

Charles John August Halberg, Jr. and Angus E. Taylor, On the spectra of linked operators .....................................

Chuan Chih Hsiung, Some integral formulas for closed hypersurfaces in Riemannian space ................................... 291

Norman D. Lane, Differentiable points of arcs in conformal n-space 301

Louis F. McAuley, A relation between perfect separability, completeness, and normality in semi-metric spaces

G. Power and D. L. Scott-Hutton, The slow shearing motion of a liquid past a semi-infinite plane .............................

A. C. Schaeffer, Entire functions

Edward Silverman, An intrinsic inequality for Lebesgue area...

Choy-Tak Taam, Asymptotic relations between systems of differential equations.

Ti Yen, Quotient algebra of a finite $A W^{*}$-algebra ... 